\title{
Every breath you take: physiology and the ecology of knowing in meditative practice
}

Jeremy Wasser(iD

Correspondence:

jwasser@cvm.tamu.edu

Department of Veterinary

Physiology \& Pharmacology, College of Veterinary Medicine \&

Biomedical Sciences, Texas A\&M University, 4466 TAMU, College

Station, TX 77843-4466, USA

\begin{abstract}
As a physiologist interested in contemplative practice and meditation I have enjoyed the opportunity of lecturing to students engaged in the study of contemplation. My pedagogic role was to expose them to some of what we know of the biological phenomena that are or may be taking place during various meditative states explicating for them the details of how the human body actually works. In the course of working with students and faculty engaged in meditation I formulated the following questions relating biological science and contemplative practice: What should a practitioner or a teacher of meditation know about basic human anatomy and physiology? Is it necessary for someone engaging in contemplative practice to understand how the human organism is actually put together and how it works? Will knowledge of how the various organs work with one another enhance one's ability to meditate or can we dispense with this information and suffer no consequences in our practice? The fundamental importance of somatic or physical phenomena in meditative practice (for example in control of breath, heart rate, or metabolism) and most people's lack of understanding of basic human anatomy and physiology led me to answer yes to all of these questions. In this paper I outline the physiological knowledge and particular insights I have found useful for enhancing a person's understanding of how we breathe, how we regulate our heart rate, and how we control our metabolic rate in 'control' or non-meditative states and the kinds of changes we might expect in a meditating subject. I link what is perhaps the fundamental principle of physiology, the concept of 'homeostasis', with the balance and integration of the body systems sought by people engaged in contemplation. Mind-body harmony or an enhanced awareness of this linkage between the mind and the body can, in my opinion, be more fully realized when coupled with an understanding of what Hippocrates called, 'the nature of the body', that is, what the body actually does and how it does it.
\end{abstract}

\section{Introduction}

What should a practitioner or a teacher of meditation or any contemplative practice know about basic human anatomy and physiology? Or should we be asking, is it necessary for someone engaging in contemplative practice to know anything about how the human organism is actually put together and how it works? Will knowledge of how the various organs work with one another enhance one's ability to meditate or can we dispense with this information and suffer no consequences in our practice?

It is, of course, true that people have been engaged in meditation and other contemplative practices for millennia without a strictly scientific understanding of human physiology.

(c) The Author(s). 2017 Open Access This article is distributed under the terms of the Creative Commons Attribution 4.0 International License (http://creativecommons.org/licenses/by/4.0/), which permits unrestricted use, distribution, and reproduction in any medium, provided you give appropriate credit to the original author(s) and the source, provide a link to the Creative Commons license, and indicate if changes were made. 
Physiological understanding is a far more modern development. There are also multiple and not mutually exclusive approaches to describing how the human organism works in addition to the "western"/empirical approach. These include ayurvedic, chi, and body energy-based ways of knowing, which tend to understand the body as indissolubly tied to the surrounding environment and cosmos. Thus our question reduces to, "Is it useful to have a western, scientifically empirical understanding of the 'nature of the body" when' engaged in meditation or when instructing others, particularly university students, on how to meditate?"

Although I am not an expert on meditation and have had only limited experiences to date as a practitioner, I am a professor of physiology and have spent years conducting research and teaching students the ways in which the human body is put together and how its parts function. New students in my undergraduate courses typically know very, very little about how we actually breathe, how we regulate our body temperatures and metabolic rates, how our hearts function, and so on. The structural and functional complexity of an organism as complicated as a human being is formidable; but people, in my experience, find learning about this intricacy fascinating and are also amazed at the ways in which all of the myriad parts of a person are integrated and work with one another. I like to say that physiology is an 'integrative' science. While we might study or teach about, for example, the heart or more broadly the cardiovascular system as a separate, distinct, anatomical entity, that part of you is connected physically to many other parts or organ systems and works with and is effected by them in complex ways. It is this connectedness that allows an organism to achieve homeostasis, the condition of balance where all of the animal's physical parts are working within their normal design parameters. We humans are built to maintain aspects like our body temperatures, breathing rates, depth of breathing, and all of our biochemical parameters within specific, acceptable limits and we have built-in control systems that monitor these variables and, when we are healthy, return them to the acceptable range when they start to deviate. I would argue here that, generally speaking, the meditative or contemplative state is also a kind of homeostasis - a state of balance - and therefore understandable in terms of basic physiological principles.

For the purposes of this article I would like to focus on three specific physiological 'systems' that I feel are particularly relevant for practitioners and teachers of meditation: the respiratory system and how we actually breathe; the cardiovascular system and how our heart actually functions; and the metabolic and thermoregulatory systems and how our body generates and processes energy. The information I discuss here will be most useful for practitioners of those kinds of meditative practices that focus on breath and breath control (pranayama), control of heart rate and blood pressure, and control of body temperature (g Tum-mo meditation). Pedagogically, my method involves posing questions about my students' own bodies and their own personal experiences with and awareness of the way their bodies work. We then explore the scientific answers to these questions together utilizing specific physical activities or 'maneuvers' that help illustrate just what is going on inside. I present here both the questions posed and the detailed answers that follow.

\section{Respiration-how we breathe}

"In this limitless world, our throat is like a swinging door. The air comes in and goes out like someone passing through a swinging door. If you think, "I breathe", the "I" is 
extra. There is no you to say "I". What we call "I" is just a swinging door which moves when we inhale and when we exhale. It just moves; that is all. When your mind is pure and calm enough to follow this movement, there is nothing: no "I", no world, no mind nor body; just a swinging door." (Roshi 1995)

Do you usually think about breathing? Are you conscious or aware of taking air in and out of your lungs? Many students of meditation and yoga appreciate the primacy of breathing and of specific breathing practices such as pranayama. Thus, when I teach respiratory physiology to either my physiology students or to students of contemplative practice I sometimes begin by asking them if they had been aware of their breathing prior to my posing the question. The answer is always 'no' and this opens the door for me to begin to explain the underlying science of why this is so.

Humans and other mammals are fortunate that here on planet Earth we have an atmosphere composed of a relatively large percentage of oxygen (a bit under 21\%) and that air, our 'respiratory medium' has a low density and is not very viscous. This means that air is easy to move around; it doesn't require a lot of energy or effort to, for example, move air in and out of our lungs. We all basically know this as, for all practical purposes, one never gets tired or fatigued from breathing (even during extreme exercise it is not the respiratory system that fatigues and limits performance). Anatomically, humans have a 'tidal' respiratory arrangement. This means that we move air into and out of our lungs through the same set of tubes (the trachea, the primary bronchi leading to each lung and the subsequent smaller and smaller bronchioles). Air is moved into these tubes and down to the inner reaches of the lung on inspiration and out again on expiration. Not all animals utilize a tidal system in this way, the classic example being fish. Fish, of course, are water-breathers and are removing the oxygen they need from that dissolved in the water. They live in an environment where the availability of oxygen is far less than in air and fish have to deal with the fact that the density and viscosity of water is quite high. This means that a fish must exert a great deal of energy moving water across their 'gas exchange surface' (gills). Fish get around this serious problem by being 'unidirectional breathers'; water is moved in one direction only across the gills, not back and forth as is the case with air-breathing animals like humans.

For us, air is moved in and out of the lungs by generating a pressure difference between the outside of the body and the inner reaches of the lung itself. This difference in pressure is referred to as a 'pressure gradient' and we call this phenomenon where a gas (or a liquid) moves from a region of higher pressure to one where the pressure is lower, 'bulk flow.' Thanks to the low density and viscosity of air, we don't need to generate a very large pressure gradient in order to move a sufficient volume of air in and out of our lungs with each breath. For an adult human, this pressure difference is very small and the volume of air moved, known as the 'tidal volume' is about 0.5 liters. How is this modest pressure gradient generated - in other words - how do we really breathe?

We need to take another look at lung and thoracic (chest cavity) anatomy in order to understand this properly. Every organ in our bodies, such as those in the chest like the lungs and heart and those in the abdomen (stomach, intestines, liver etc.) are covered on their outer surfaces by a thin sheet of connective tissue known as the 'visceral pleura.' There is a second connective tissue sheet on the inner side of the chest and abdomen. The entire inside of your torso is lined by this second, thin sheet of tissue that 
is known as the 'parietal pleura.' In the chest, there is a small space between the lungs and the chest wall, thus between the visceral and parietal pleurae. We call this the 'interpleural space' and it is filled with fluid. This liquid acts to connect or 'couple' the lung to the chest wall. In other words, in a healthy and functioning human respiratory system, one could say the lung is 'stuck' to the inside of the chest and when the chest moves - either in or out with in-breath or out-breath - so does the lung.

In order to inspire, we increase the volume of the chest, thereby increasing the volume of the lung; since the lung is mechanically coupled or stuck to the chest wall thanks to the liquid in the pleural space. This increase in volume is exactly what we wish to do in order to generate a lower pressure in the lung than outside the body. Why lower? Now we have to introduce a little bit of basic physics to our discussion. Boyle's Law (named for the $17^{\text {th }}$ century Anglo-Irish chemist, physicist, and natural philosopher) states that in a closed system (a sealed beaker or our lungs) an increase in volume results in a decrease in pressure of the system and a decrease in volume results in an increase in pressure. This is due to the forces being generated by the moving gas molecules contained within the system. For example, the more room these molecules have to move around in, the less likely they are to collide with one another or with the walls of the container and it is these collisions that are responsible for creating the pressure. So, when you increase the volume of the lung (by increasing the volume of the chest), you decrease the pressure inside the lung. Gas then moves from a region of higher pressure (the environment) to a region of lower pressue (the inside of the lung). This is in-breath or inspiration. The opposite occurs during out-breath (expiration) where we decrease the volume of the chest and thus the lung, increasing the pressure inside compared to the room pressure and so, out comes the gas! We do not suck or push air into our lungs - the lungs are not muscular organs - they are filled and emptied via the physical principle of Boyle's Law described above and the fact that they are mechanically coupled to the chest wall and must move (change volume) as the volume of the chest is changed.

How do we change the volume of the chest in order to breathe? In order to inspire, we must contract one and only one skeletal muscle, a very special one called the diaphragm. The diaphragm lies between the thorax and abdomen separating them completely into two body compartments. It is a sheet-like, dome-shaped muscle and is not attached to the lungs directly. Rather, when we wish to inspire, the diaphragm contracts (shortens) causing it to flatten out and move downward towards the abdomen. This contraction also moves the ribs outwards slightly. In other words, a contraction of the diaphragm increases the volume of the chest, and thus the lungs, decreasing the pressure and allowing air to flow from where the pressure is relatively higher (the room) to where it is now relatively lower. On expiration, the diaphragm relaxes and returns to its original higher and flatter position resulting in a decrease in chest and lung volumes, an increase in pressure in the lung and the consequent movement of air out of the lung and back into the room.

Thus, in physiological terms, inspiration is an active process because it requires the contraction of the diaphragm whereas expiration is considered a passive process as it relies solely on the elastic properties of the lung and chest wall. In this context, you may think of the lung and chest as a balloon that is filled on inspiration. On expiration, air flows out of the body and these structures return to their original shapes/sizes the 
way a balloon deflates or the way a spring or rubber band returns to its original shape when the tension is released.

Now there are some additional skeletal muscles in the chest, neck and abdomen that can play a role in breathing but these are all 'accessory' muscles of either inspiration or expiration. They are not required for a person to adequately breathe and are brought into play only when we make the demand on our respiratory systems to 'breathe more' by which I mean move more air in and out of the system every minute (for example during exercise).

\section{How long can you hold your breath?}

"Yogiraja Vaidyaraja, the so-called "burying yogi"... often spent two or three days buried in a box several feet underground to demonstrate his devotion to his followers. For the purposes of Green's test, the yogi was sealed in the lotus position in a completely airtight cube... After nearly eight hours in the airless box, the yogi signaled his desire to be let out, complaining that he had received three electric shocks from the equipment... readouts showed that his breath rate had dropped to less than four breaths per minute,..." (Robbins 2008)

How long can you hold your breath? During a breath hold, what causes you to finally give up and resume breathing? Although there are reports of yogis being buried alive and surviving for long periods of time (days or weeks) these are mostly anecdotal and may not represent actual occurrences. I like to show my students a film clip from 1929 in British-controlled India of a yogi buried for an hour and a half, after which he is disinterred and is undamaged by the experience. I then ask students to hold their breath as long as possible. Prior to the breath-hold, they are free to hyperventilate a bit if they wish, breathing in and out deeply five or six times. Most untrained humans can only hold their breath for about a minute or so after which the urge to breathe (air hunger or dyspnea) becomes so overwhelming that they must resume breathing.

The obvious next question I ask is, 'what exactly forced you to resume breathing'? Many students answer that they must have run out of oxygen and their lack of this vital gas forced them to stop the breath-hold maneuver and start ventilating the lung with air again. This is a reasonable answer but is, in fact, wrong. At the end of a period of voluntary apnea (voluntary breath-hold) there has actually been no significant change on the amount of oxygen in your blood (98\% of which is bound to the hemoglobin protein in the red blood cells). In other words, you have plenty of oxygen on board to provide all the cells of your body with what they need to produce energy and keep you alive. The 'break point of voluntary apnea' has nothing to do with oxygen and everything to do with carbon dioxide $\left(\mathrm{CO}_{2}\right)$ which is busily building up during even a rather short period of breath-hold and it is this increase in $\mathrm{CO}_{2}$ that forces you to breathe again. The body's level of $\mathrm{CO}_{2}$ is the controlling parameter in humans under almost all physiological and environmental conditions and it is $\mathrm{CO}_{2}$ that regulates breathing. Students naturally want to know where this carbon dioxide is coming from since there is essentially none in the air we breathe. After all, along with the approximate $21 \%$ oxygen, our earthly atmosphere contains about $79 \%$ nitrogen and only $0.03 \%$ carbon dioxide. The answer then? We make $\mathrm{CO}_{2}$ as an end product of our body's metabolic 
chemistry and the regulatory control of carbon dioxide levels in the body is handled for us by a control center made up of a group of nerve cells located in the brain that monitors blood $\mathrm{CO}_{2}$ levels and commands us to breathe more or less in order to keep that level where it ought to be. When you breathe less or, more dramatically, hold your breath, $\mathrm{CO}_{2}$ builds up in the body and blood, while the opposite occurs when you breathe more (hyperventilate).

At this point I also introduce my students to the sport of breath-hold diving and point out that the world record for 'static apnea' (holding one's breath while facedown and motionless in a pool) is over 20 minutes! This is quite extraordinary and the competitors increase their abilities to breath-hold by prior hyperventilating on pure oxygen. This decreases the amount of carbon dioxide in their bodies to below normal levels thereby increasing the time it takes to build up to a level that will force you to breathe. They also take advantage of meditative/relaxation techniques to decrease their metabolic rates, which will also slow the rate of carbon dioxide production.

Meditators have also demonstrated the ability to lower their metabolic rates significantly. In one study, oxygen consumption (a measure of metabolic rate) during meditation in three subjects showed a 16\% decrease within less than 30 minutes (Wallace and Benson 1972). When we speak of metabolism or metabolic rate what we are really considering is the sum total of all of the chemical reactions taking place in every cell throughout the body. These chemical reactions are governed by physical laws including those of thermodynamics and include reactions that produce heat (exergonic reactions) and those that consume heat (endergonic reactions). It simply isn't possible to measure the thermodynamic changes in every chemical reaction in a living being and what we do instead is consider overall metabolism as the body's heat production, which is something that can be physically measured in the laboratory. This method, known as direct calorimetry, is also technically demanding and most metabolic physiologists measure oxygen consumption or carbon dioxide production instead (indirect calorimetry). There is a direct correlation between the amount of oxygen consumed by a person per minute and the amount of heat they are producing. This 'caloric equivalent of oxygen' varies depending on the type of substrate (nutrient) being metabolized (carbohydrate, fat, protein, or a mixture) so you also have to know this in order to accurately calculate a metabolic rate based on oxygen consumption.

Chemical reactions (and hence metabolic rates) are also quite sensitive to changes in temperature; in the case of a human being, body temperature. As temperature goes up, so does metabolic rate and as temperature decreases we see a corresponding decrease in metabolism. Humans (even meditating ones), as a rule, do not experience large swings in core body temperature (that is the temperature deep within the chest or abdomen) and the decrease in metabolism seen in Wallace and Benson's (1972) study cannot be attributed to this temperature effect. Naturally, the lower one can decrease metabolism, the slower the utilization of oxygen and, importantly for humans, the slower the production and buildup of carbon dioxide in the body. This depressed metabolic or 'hypometabolic' state increases the time it takes for $\mathrm{CO}_{2}$ to reach the point where a person must stop breath-holding (the break-point of voluntary apnea).

At this point in my lectures I like to introduce my students to some non-human animals that are particularly adept at entering a controlled, hypometablic state, and as a consequence are able to breath-hold for an extraordinarily long time. Seals and whales, 
mammals that are highly adapted for diving, exhibit a suite of anatomical and physiological adaptations that optimize their ability to reduce overall oxygen consumption (and carbon dioxide production) and stay underwater for, in some cases, hours. I also teach them about the truly remarkable ability of some turtles to reduce their metabolic rates to $10 \%$ of their oxygen-breathing levels when forced underwater with no access to air (Jackson 1968). They do this with no change in their body temperatures. The phenomenon is solely a function of making oxygen unavailable and allows these animals to effectively (although not indefinitely) live without oxygen.

The breath-hold exercise also allows me to begin to discuss the control of ventilation (breathing) in humans that is quite unusual compared to say, control of heart rate or body temperature. The students have just demonstrated to themselves that they can voluntarily and easily control the way they breathe - up to a point. In other words, ventilation is under both voluntary and involuntary regulatory control. We don't usually think about breathing at all and we breathe successfully when we are asleep or otherwise unconscious. Yet we can hold our breath for a short while or alter how deep each breath is (change the tidal volume) or increase or decrease the number of breaths we take each minute (change the respiratory frequency). Ultimately, however, the involuntary system will 'win' and reestablish control over the way in which we breathe.

When you meditate and bring conscious awareness to your breathing, you are activating the neural pathways associated with conscious control and overriding those that typically cause your breathing pattern (depth of breath and rate) to be a completely unconscious process. In general, our bodies 'know' how much we should breathe in order to meet the metabolic demands of the moment (need for oxygen and the need to remove the excess carbon dioxide we are producing). An altered breathing pattern (for example, breath of fire in Kundalini yoga) is something that you are imposing on the body. This may account for the difficulty in correctly and effectively engaging in various breathing regimens while meditating. It also explains the fact that you cannot maintain an imposed, voluntary, breathing pattern indefinitely. Over time, you will ultimately revert back to the unconscious and optimal pattern (optimal in terms of metabolism, not necessarily in terms of your meditative goals). As in all things, practice improves the ability to establish and maintain an imposed voluntary breathing pattern but you will always have to engage the thinking and planning portions of the brain located in the cerebral cortex to continue breathing this way.

\section{Cardiovascular physiology-how our heart beats}

"Having visited there (Mysore) before, I once witnessed a demonstration given by the pupils followed by an extraordinary feat by Shri Krishnamachacharya, the teacher. He lay on the floor and then proceeded to stop his heart beat for several minutes much to the astonishment of several physicians who had come with their stop watches and stethoscopes. 'I would have pronounced him dead', said a German doctor after the examination." (Devi 1967)

Is it possible to consciously control heart rate? Heart rate and related cardiovascular parameters such as blood pressure or stroke volume (the volume of blood pumped from the heart with each beat) are, from a physiological perspective, not considered to 
be things that we can control consciously. The heart itself is quite extraordinary in that it exhibits the property of 'autorhythmicity'; that is to say it is capable of beating on its own without any input or signaling from the nervous system and in the absence of any signaling from chemicals in the blood. This built-in or intrinsic heart rate is determined by the beating frequency of a specific anatomical region of the heart, the sinoatrial node, located in the upper right-hand corner of the right atrium (right upper heart chamber). The cells of the sinoatrial node are capable of spontaneous depolarization, which means that they rhythmically generate an electrical signal that then spreads through the rest of the heart driving the overall heart rate.

In humans, the built-in rate of signaling by the sinoatrial node is about 100 beats per minute whereas the normal human resting heart rate is typically somewhere between 50 and 80 beats per minute. What is responsible for this difference? While the heart is capable of beating without any connection to the nervous system, in our bodies nerves do connect to the heart and influence its function. The nerves that leave the brain and spinal cord and connect to our various organs, skeletal muscles and other body parts can be divided into the 'somatic' and the 'autonomic' nervous systems. Somatic nerves are those that leave the spinal cord and connect to skeletal muscles allowing them to contract and generate force. The autonomic nervous system is itself divided into two parts (divisions), the sympathetic and the parasympathetic and leaves the brain and spinal cord to innervate our internal organs and glands. Most of our organs are 'dually innervated.' In other words, they are connected to and influenced by nerves from both the sympathetic and parasympathetic divisions of the autonomic nervous system. In the case of the heart, the vagus nerve leaves the brain and sends branches to the sinoatrial nodal cells. The vagus nerve is part of the parasympathetic nervous system and its role in heart function is to slow down the intrinsic rate of contraction of the sinoatrial cells and therefore the heart rate as a whole. Healthy humans have a high degree of 'parasympathetic tone,' i.e. relatively lots of signaling from the vagus nerve compared to the sympathetic nerves that also connect to the heart and when stimulated, increase heart rate. This parasympathetic tone is responsible for our resting heart rates being about 70 beats per minute rather than the faster intrinsic rate of the sinoatrial node itself.

Reports of yogis being able to actually stop their hearts from beating altogether have not been scientifically validated (see Wenger et al. 2002). However, it does appear possible to modulate heart rate and blood pressure to some extent via relaxation methods and meditative techniques. In physiological terms what must be happening here is a change in the ratio of sympathetic (stimulatory, increasing rate) versus parasympathetic (inhibitory, decreasing rate) tone (activity) throughout the body. This will have a direct effect on the activity of the sinoatrial node and therefore the entire heart (Amihai and Kozhevnikov 2015).

How exactly these mental disciplines increase parasympathetic tone is less clear although there are nervous connections between higher brain centers in the cerebral cortex and those parts of the brain (hypothalamus and brain stem) responsible for the autonomic (unconscious) functioning of the heart and other organs. In my teaching I use the example of heart rate modulation during meditation as a way to explain not just how our hearts actually beat, but also to introduce elements of neuroanatomy and neurophysiology, particularly the way in which neural signaling from the cortex (higher brain centers) can influence what are normally automatic and unconscious aspects of organ function. 


\section{Thermoregulation and metabolism - how we control body temperature and metabolism}

'During the practice of $g$ Tum-mo yoga, 'prana' (literally, 'wind' or 'air') is withdrawn from the scattered condition of normal consciousness and is made to enter into the 'central channel' inside the body. Then, through the alleged dissolution of these winds in the central channel, the 'internal heat' is ignited. The physiological changes are, therefore, a by-product of a religious practice." (Benson et al. 1982).

Can you consciously control and manipulate your body temperature? Humans, like most mammals are 'homeotherms.' This means that we closely and carefully regulate our internal, deep body temperatures (core temperatures) around a specific temperature and that we engage physiological control systems to make sure this temperature never varies very much. For an adult human, this core body temperature is about $37^{\circ} \mathrm{C}\left(98.6^{\circ} \mathrm{F}\right)$ and in a healthy individual will only range by 1 or 2 degrees above or below. I ask my students to consider what we mean when we say 'body temperature' for in fact, there are many different temperatures found at different places in our bodies. Along with deep core (maintained at around $37^{\circ} \mathrm{C}$ ) the temperature of our skin varies depending on environmental temperatures and is influenced as part of the regulatory control we use to maintain core temperature.

I often have my students consider what is happening in their bodies when they are heating up, either because of a high environmental temperature or from exercise (where the heat is coming from an increase in metabolism). They know that when they are getting hot, they will start to sweat and also notice a change in the color of their skin. In order to eliminate extra heat we change the amount of blood flow directed towards the surface of our bodies and we see this as a reddening or flush (particularly obvious in fair-skinned individuals but occurring in everyone). Cutaneous (skin) blood flow can increase enormously as a fraction of total blood flow from the heart when we are heat stressed and as this warm blood moves to the skin it carries the heat along with it. If the environmental conditions are suitable, we can then 'dump' heat from our warm skin to the environment. This vascular (blood vessel and blood flow) response to a heat stress complements sweating as the two main mechanisms for heat loss in humans.

The increase in skin temperatures of some master practitioners of g Tum-mo meditation (Benson et al. 1982) suggests that they are somehow capable of manipulating blood flow to the skin. This is not something that is normally considered to be under voluntary control. An alternative hypothesis is that these masters are increasing the metabolic rate of those regions of their body (fingers and toes, typically) that are heating up. Remember that heat occurs in our bodies from either external (environmental) or internal (metabolic) sources. To the best of my knowledge no direct measurements have yet been made of either local skin blood flow or regional metabolic rate during g Tum-mo meditation. Nor do we have a scientific hypothesis for how these monks would be capable of manipulating physiological systems (blood flow and metabolism) generally considered to be completely automatic and unconscious. 


\section{Conclusion}

"Some offer their out-flowing breath into the breath that flows in; and the in-flowing breath into the breath that flows out; they aim at Pranayama, breath-harmony, and the flow of their breath is in peace." The Bhagavad Gita

Students and teachers of meditation are already believers in the reality and importance of these practices for physical health and psychological well-being and they are justified in this belief from an ever-growing body of evidence-based studies. What they lack, however, in many instances is an awareness and appreciation of the anatomy and physiology that underlies what is (or in some cases may be) taking place during meditative practice and I strongly believe they can benefit from having their questions addressed in terms of the actual physiological mechanisms at work during meditation. My students often express what can only be called astonishment and a true sense of wonder when taught about how their bodies and those of other animals actually work. Since some of what I teach them about breathing, metabolism, heart beat, body temperature, and other physiological systems is often counterintuitive and flies in the face of what they have always believed, the impact of this new knowledge is dramatic. I cannot say with assurance that a sophisticated understanding of physiology necessarily improves one's ability to enter contemplative states and practice meditation. However, if the meditator's goal is increased self-knowledge and deeper self-awareness, then a more precise and analytical appreciation of what is physically going on inside the body can provide another pathway into the kind of personal exploration that underlies all contemplative activities.

I also teach students in traditional medicine or biomedical science programs who are equally unaware of how their bodies actually work and who are also unfamiliar or skeptical of the benefit of meditative practices (and any other non-western health or medical tradition). I want to send these students into the medical community with an open mind about these alternative possibilities and to seriously consider them as potential adjunct therapies or lifestyle adjustments as they begin their careers in medicine.

I want my students, whether of medicine or meditation, to come away from these lectures and exercises with an increased understanding of the 'nature of the body'. I also want them to experience excitement and interest in learning more about what is truly going on inside of each of us. That a master of meditation should also be a master of physiology makes perfect sense to me. The ultimate goal of both of these disciplines is to further the understanding of the total human being as an integrated organism functioning in harmony with both its internal and external environments.

\section{Acknowledgement}

I would like to acknowledge Professor Jane Brucker for our many conversations on contemplative practice and for the opportunity to present elements of the relevant anatomy and physiology to her students.

Competing interest

The author declares that he/she has no competing interests.

Received: 14 October 2016 Accepted: 7 December 2016

Published online: 17 January 2017

References

Amihai, Ido and Maria Kozhenikov. 2015. The Influence of Buddhist Meditation Traditions in the Autonomic System and Attention. BioMed Research International. http://dx.doi.org/10.1155/2015/731579. Accessed 27 Sept 2016. 
Benson, Herbert, John W. Lehmann, M.S. Malhotra, Ralph F. Goldman, Jeffrey Hopkins, and Mark D. Epstein. 1982. Body temperature changes during the practice of g tum-mo yoga. Nature 295: 234-236.

Devi, Indra. 1967. Yoga the Technique of Health and Happiness. Bozeman: Jaico.

Jackson, D.C. 1968. Metabolic depression and oxygen depletion in the diving turtle. Journal of Applied Psychology 24(4): 503-509.

Robbins, Jim. 2008. A Symphony in the Brain: The Evolution of the New Brain Wave Biofeedback. New York: Grove Press.

Roshi, Shunryu S. 1995. Breathing. Tricycle Magazine. http://tricycle.org/magazine/breathing-4/. Accessed 27 Sept 2016.

Wallace, Robert K, and Herbert Benson. 1972. The physiology of meditation. The Scientific American 226(2): 84-90. http://tricycle.org/magazine/breathing-4/. Accessed 27 Sept 2016.

Wenger, MA, BK Bagchi, and BK Anand. 2002. Experiments in India on "Voluntary" Control of the Heart and Pulse. Circulation 24: 1319-1325

Submit your manuscript to a SpringerOpen ${ }^{\circ}$ journal and benefit from:

- Convenient online submission

- Rigorous peer review

- Immediate publication on acceptance

- Open access: articles freely available online

- High visibility within the field

- Retaining the copyright to your article

Submit your next manuscript at $>$ springeropen.com 